\title{
HAMILTONIAN-CONNECTED GRAPHS AND THEIR STRONG CLOSURES
}

\author{
PAK-KEN WONG \\ Department of Mathematics and Computer Science \\ Seton Hall University \\ South Orange, NJ 07079
}

(Received September 15, 1993 and in revised form November 2, 1993)

\begin{abstract}
Let $G$ be a simple graph of order at least three. We show that $G$ is Hamiltonianconnected if and only if its strong closure is Hamiltonian-connected. We also give an efficient algorithm to compute the strong closure of $G$.
\end{abstract}

KEY WORDS AND PHRASES. Hamiltonian-connected graph, strong closure, degree sequence. 1991 AMS SUBJECT CLASSIFICATION CODE(S). 05C45, 05C99.

\section{INTRODUCTION.}

Let $G=(V, E)$ be a simple graph, $n=|V|(\geq 3)$ and $m=|E| . \quad G$ is called Hamiltonianconnected if every two vertices of $G$ are connected by a Hamiltonian path. If $G$ is Hamiltonianconnected and $n \geq 4$, then $m \geq \frac{1}{2}(3 n+1)$ (see [2], p. 61).

In this paper, we define the strong closure $s c(G)$ of a simple graph $G$. We also show that $G$ is Hamiltonian-connected if and only if its strong closure $s c(G)$ is Hamiltonian-connected (Theorem 2.3). It follows immediately that if $s c(G)$ is a complete graph, then $G$ is Hamiltonianconnected (Corollary 2.4). As in the case of Hamiltonian graphs, there is no characterization of Hamiltonian-connected graphs. If we compute the strong closure of $G$ and find it is complete, then $G$ is Hamiltonian-connected. As another application, a result of $O$. Ore also follows from Corollary 2.4 (see Corollary 2.5).

In section 3, we give an efficient algorithm to compute the strong closure $s c(G)$ of any simple graph $G$. This algorithm can be executed in $O(n|K|)$ time, where $\left|K^{\prime}\right|=\frac{1}{2}\left(n^{2}-n-2 m\right)$.

\section{HAMILTONIAN-CONNECTED GRAPHS.}

For each vertex $v$ of $G$, let $D(v)=\{u \in V(G): u$ is adjacent to $v\}$. Then $d(v)=|D(v)|$ is the degree of $v$ in $G$.

We have the main result of this paper.

THEOREM 2.1. Suppose that $u$ is not adjacent to $v$ in $G$ and $d(u)+d(v) \geq n+1$. Then $G$ is Hamiltonian-connected if and only if $G+(u, v)$ is Hamiltonian-connected.

PROOF. Suppose that $G+(u, v)$ is Hamiltonian-connected, but $G$ is not. Since $G$ is not Hamiltonian-connected, there exist two vertices $x$ and $y$ such that there is no Hamiltonian $x-y$ path in $G$. Since $G+(u, v)$ is Hamiltonian-connected, there is a Hamiltonian $u-v$ path in $G+(u, v)$ and hence in $G$. Therefore it follows that $(x, y) \neq(u, v)$. Let $P=\left\{w_{1}, w_{2}, \cdots, w_{n}\right\}$ be a Hamiltonian $x-y$ path in $G+(u, v)$, where $x=w_{1}$ and $y=w_{n}$. 
CASE 1. Assume that $x \neq u$ and $y \neq v$. Since $P$ is a Hamiltonian $x-y$ path in $G+(u, v)$ and $P$ is not a Hamiltonian $x-y$ path in $G,(u, v)$ must be an edge of $P$ in $G+(u, v)$. Therefore $u=w_{k}$ and $v=w_{k+1}$ for some $1<k<n-1 . \quad(k \neq n-1 ;$ for otherwise $k+1=n$ and $\left.v=w_{n}=y\right)$. Since $(u, v)$ is not an edge of $G, u, v \notin D(u)$ and $u, v \notin D(v)$. Suppose $w_{t} \in D(u)$, where $t \neq k-1$ and $t \neq n$. \Since $u\left(=w_{k}\right)$ and $v\left(=w_{k+1}\right)$ are not in $D(u)$, it follows that $t \neq k$ and $t \neq k+1$. We show that $w_{t+1} \notin D(v)$. Suppose that this is not true, then $w_{t+1}$ is adjacent to $v$. If $t<k-1$, then the path $\left(=w_{1}\right), w_{2}, \cdots, w_{t}, w_{k}(=u), w_{k-1}, w_{k-2}, \cdots, w_{t+1}$, $v\left(=w_{k+1}\right), w_{k+2}, \cdots, y\left(=w_{n}\right)$ is a Hamiltonian $x-y$ path in $G$. If $t>k+1$, then the path $x\left(=w_{1}\right), w_{2}, \cdots, w_{k}(=u), w_{t}, w_{t-1}, \cdots, w_{k+1}(=v), w_{t+1}, w_{t+2}, \cdots, y\left(=w_{n}\right)$ is a Hamiltonian $x-y$ path in $G$. This is impossible. Therefore, $w_{t+1} \notin D(v)$. Since $t \neq k-1$ and $t \neq n$, it follows that there are at least $d(u)-2$ vertices to which $v$ is not adjacent. Since $u, v \notin D(v)$, we have

$$
d(v) \leq(n-2)-(d(u)-2)=n-d(u)
$$

Therefore $d(u)+d(v) \leq n$, which is a contradiction. Therefore Case 1 is impossible.

CASE 2. Assume that $v=y\left(=w_{n}\right)$. Since $(x, y) \neq(u, v)$, it follows that $u \neq x$ and so $u=w_{n-1}$. Let $w_{t} \in D(u)$, where $t \neq n-2$. Then by the same argument as in Case 1, $w_{t+1} \notin D(v)$. Hence

$$
d(v) \leq(n-2)-(d(u)-1)=n-d(u)-1
$$

and so $d(u)+d(v) \leq n-1$, which is impossible. Therefore $G$ is Hamiltonian-connected. The converse of the theorem is clearly true. This completes the proof of the theorem.

Theorem 2.1 motivates the following definition.

The strong closure of $G$ is the graph obtained from $G$ by recursively joining pairs of nonadjacent vertices whose degree sum is at least $n+1$ until no such pair remains. We denote the strong closure of $G$ by $s c(G)$.

REMARK. The closure $c(G)$ of $G$ is defined and studied in [2] and [4]. It is useful in the study of Hamiltonian graphs. The definition of $s c(G)$ is similar to that of $c(G)$.

LEMMA 2.2. $s c(G)$ is well-defined.

PROOF. This follows from the proof of ([2], p. 56, Lemma 4.4.2).

THEOREM 2.3. A graph is Hamiltonian-connected if and only if its strong closure is Hamiltonian-connected.

PROOF. This follows immediately from Theorem 2.1 and Lemma 2.2.

Theorem 2.3 gives some interesting results.

COROLLARY 2.4. If $s c(G)$ is a complete graph, then $G$ is Hamiltonian-connected.

PROOF. If $s c(G)$ is complete, then it is Hamiltonian-connected and so by Theorem 2.3, $G$ is also Hamiltonian-connected.

The following result was obtained by $O$. Ore (see [1], p. 136, Theorem 11.3 or [5]).

COROLLARY 2.5. If $d(u)+d(v) \geq n+1$ for every pair of nonadjacent vertices $u$ and $v$, then $G$ is Hamiltonian-connected.

PROOF. Since $d(u)+d(v) \geq n+1$ for every pair of nonadjacent vertices $u$ and $v$, it follows that $S c(G)$ is a complete graph. Therefore by Corollary $2.4, G$ is Hamiltonian-connected.

If $G$ has vertices $v_{1}, v_{2}, \cdots, v_{n}$, the sequence $\left(d\left(v_{1}\right), d\left(v_{2}\right), \cdots, d\left(v_{n}\right)\right)$ is called a degree sequence of $G$. The following result is similar to a result obtained by Chvátal (see [2], p 57, Theorem 4.5). 
COROLLARY 2.6. Let $\left(d_{1}, d_{2}, \cdots, d_{n}\right)$ be a degree sequence of $G$ such that $d_{1} \leq d_{2} \leq \cdots \leq d_{n}$. Suppose that there is no value of $p$ less than $\frac{1}{2}(n+1)$ for which $d_{p-1} \leq p$ and $d_{n-p}<n-(p-1)$. Then $G$ is Hamiltonian-connected.

PROOF. By a similar argument as in the proof of ([2], p. 57, Theorem 4.5), we can show that $s c(G)$ is a complete graph. Therefore by Corollary $2.4, G$ is Hamiltonian-connected.

\section{AN ALGORITHM FOR FINDING STRONG CLOSURE.}

In this section, we give an algorithm to find $s c(G)$. Let $V(G)=\left\{u_{1}, u_{2}, \cdots, u_{n}\right\}$.

STEP 1. For $1 \leq i<j \leq n$, let

$$
f(i, j)= \begin{cases}d\left(u_{\imath}\right)+d\left(v_{\jmath}\right), & \text { if } u_{\imath} \notin D\left(v_{\jmath}\right) \\ 0 & , \text { if } u_{\imath} \in D\left(v_{\jmath}\right)\end{cases}
$$

STEP 2. Choose $f(I, J)=\max \{f(i, j): 1 \leq i<j \leq n\}$.

If $f(I, J)<n+1$, then go to Step 4.

STEP 3. $f(I, J) \leftarrow 0$.

If $f(p, I) \neq 0$, then $f(p, I) \leftarrow f(p, I)+1(1 \leq p<I)$.

If $f(I, p) \neq 0$, then $f(I, p) \leftarrow f(I, p)+1(I<p \leq n)$.

If $f(q, J) \neq 0$, then $f(q, J) \leftarrow f(q, J)+1(1 \leq q<J)$.

If $f(J, q) \neq 0$, then $f(J, q) \leftarrow f(J, q)+1(J<q \leq n)$.

Go to Step 2.

STEP 4. Form $s c(G)$ by joining $u_{i}$ to $u_{\jmath}$, if $f(i, \jmath)=0(1 \leq i<j \leq n)$.

Let $G$ be represented by an adjacency matrix. Steps 1 and 4 can be implemented in $O\left(n^{2}\right)$ time. Clearly, Step 3 runs in $O(n)$ time. Let $K=\{(i, j): f(i, j) \neq 0 \quad 1 \leq i<j \leq n\}$. Then

$$
|K|=1+2+\cdots+(n-1)-m=\frac{1}{2}\left(n^{2}-n-2 m\right) .
$$

By using $F$-heaps data structure [3], find $\max \{f(i, j)\}$ takes $O\left(\log _{2}|K|\right)=O\left(\log _{2} n\right)$ time. Hence Steps 2 and 3 take $O\left(|K|\left(n+\log _{2} n\right)\right)=O(n|K|)$. Thus overall we have an $O(n|K|)$ algorithm.

LEMMA 3.1. If $n \geq 4$ and $d(u) \leq 2$ in $G$, then $d(u) \leq 2$ in $s c(G)$.

PROOF. Let $v$ be a vertex of $G$ which is not adjacent to $u$. Then $d(v) \leq n-2$. Hence $d(u)+d(v) \leq 2+(n-2)=n$ and so Lemma 3.1 is true.

Lemma 3.1 allows us not to consider $u$ in the computation of $s c(G)$ if $d(u) \leq 2$ in $G$.

\section{REFERENCES}

1. BEHZAD, M. and CHARTRAND, G., Introduction to the Theory of Graphs, Allyn and Bacon Inc., Boston, 1971.

2. BONDY, J.A. and MURTY, U.S.R., Graph Theory with Applications, North-Holland, New York, 1976.

3. FREDMAN, M.L. and TARJAN, R.E., Fibonacci heaps and their uses in improved network optimization algorithms, J. ACM 34 (1987), 596-615.

4. GONDRAN, M. and MINOUX, M., Graph and Algorithms, Wiley, New York, 1984.

5. ORE, O., Hamilton connected graphs, J. Math. Pures. Appl. 42 (1963), 21-27.

6. WONG, P.K., On the closure of a graph with cut vertices, Ars Combinatoria 35 (1993), 253-256. 


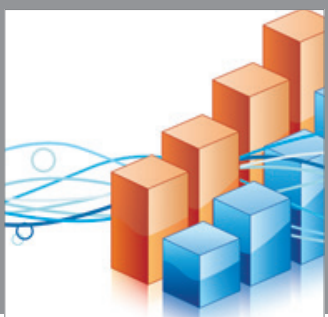

Advances in

Operations Research

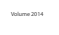

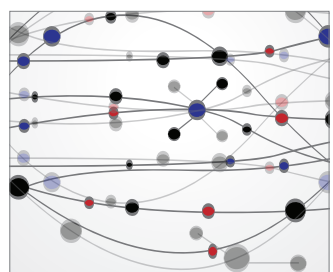

\section{The Scientific} World Journal
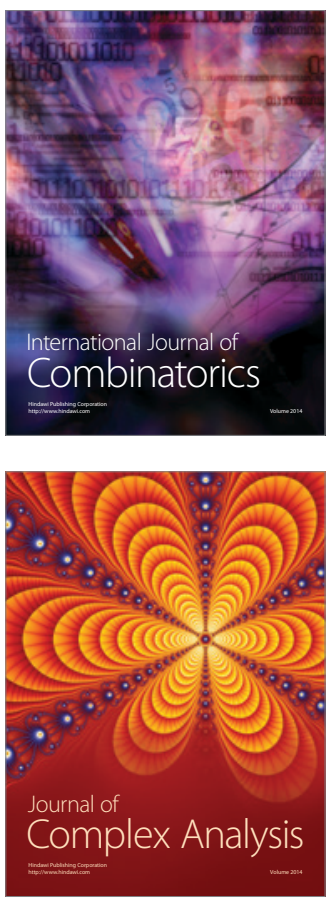

International Journal of

Mathematics and

Mathematical

Sciences
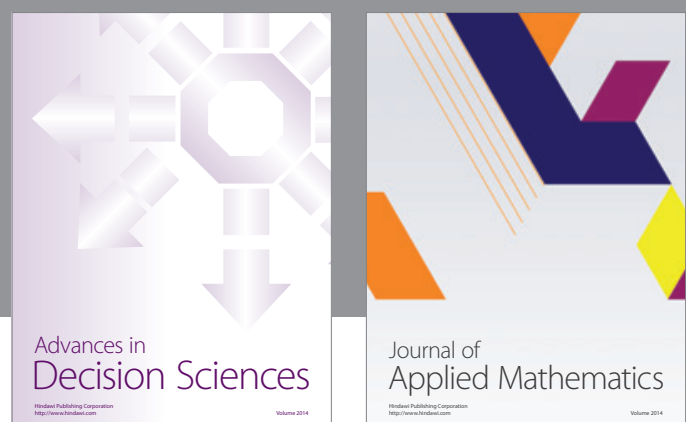

Journal of

Applied Mathematics
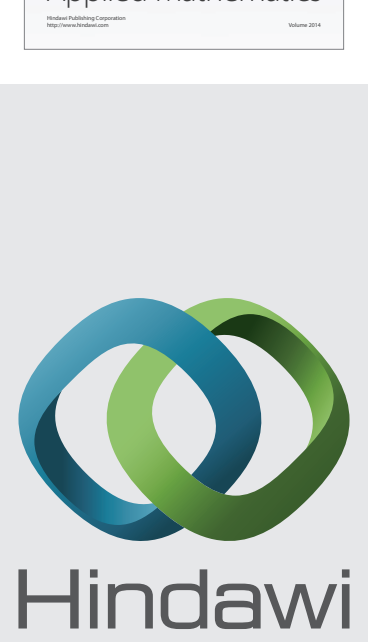

Submit your manuscripts at http://www.hindawi.com
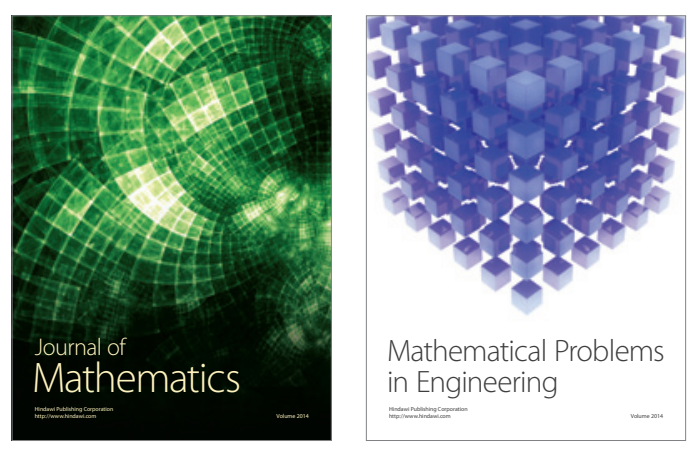

Mathematical Problems in Engineering
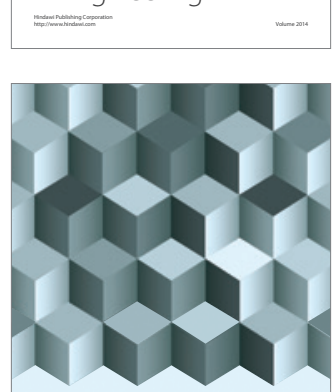

Journal of

Function Spaces
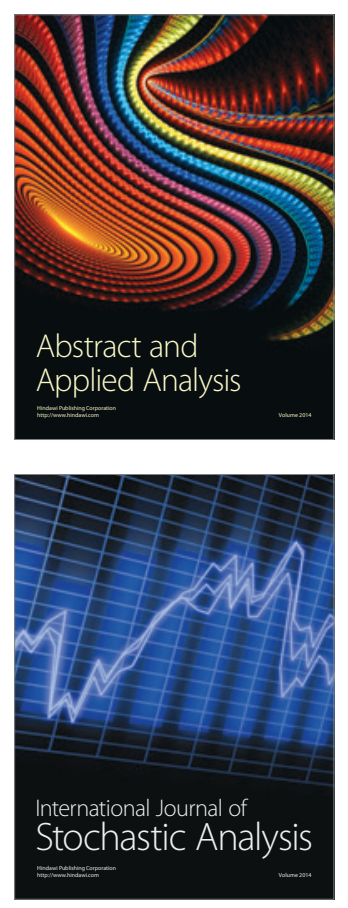

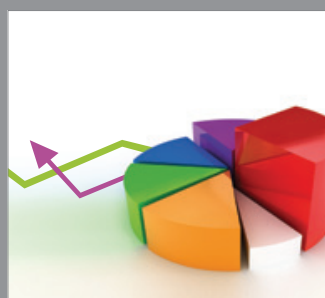

ournal of

Probability and Statistics

Promensencen
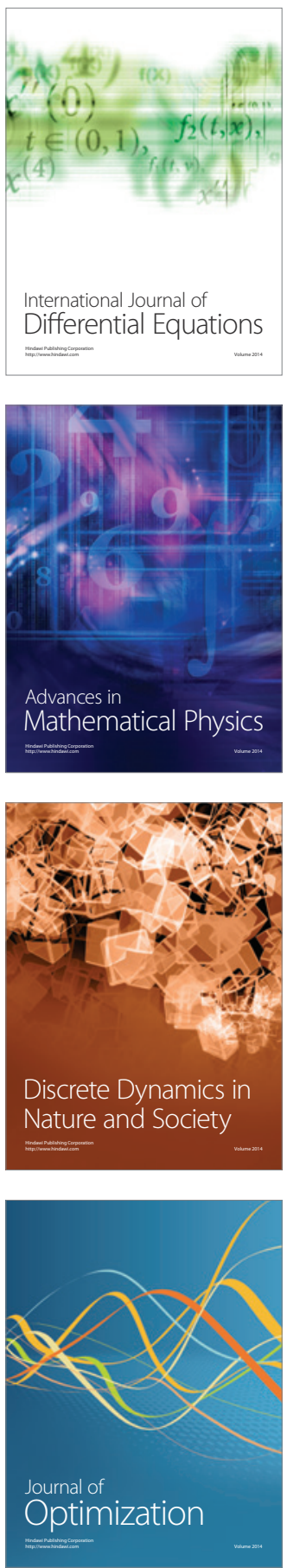